Revue des patrimoines

\title{
Les bâtiments ruraux dans leur environnement en pays de bocage et en pays d'openfield
}

\section{Pierre Brunet}

\section{(2) OpenEdition}

\section{Journals}

Édition électronique

URL : http://journals.openedition.org/insitu/2545

DOI : 10.4000/insitu.2545

ISSN : $1630-7305$

\section{Éditeur}

Ministère de la culture

Référence électronique

Pierre Brunet, «Les bâtiments ruraux dans leur environnement en pays de bocage et en pays d'openfield », In Situ [En ligne], 7 | 2006, mis en ligne le 27 février 2012, consulté le 02 mai 2019. URL http://journals.openedition.org/insitu/2545; DOI : 10.4000/insitu.2545

Ce document a été généré automatiquement le 2 mai 2019.

\section{(c) (i) (9)}

In Situ Revues des patrimoines est mis à disposition selon les termes de la licence Creative Commons Attribution - Pas d'Utilisation Commerciale - Pas de Modification 4.0 International. 


\title{
Les bâtiments ruraux dans leur environnement en pays de bocage et en pays d'openfield
}

\author{
Pierre Brunet
}

1 Le contenu d'un inventaire des bâtiments ruraux dépasse les églises, chapelles, châteaux et manoirs protégés. Il englobe les bâtiments des exploitations agricoles (logis et bâtiments d'usage), les maisons des autres habitants de la campagne (artisans, commerçants, journaliers et ouvriers agricoles), des bâtiments de service collectif (lavoirs, moulins, fours à pain, etc.). De telles recensions ont déjà été réalisées dans de nombreux cantons par l'Inventaire, tels ceux de Vimoutiers et de Ryes en BasseNormandie.

2 Cependant ces édifices ne doivent pas être considérés isolément. Ils ne sont pas disposés arbitrairement les uns par rapport aux autres, ni sans relations avec l'aménagement de l'espace qui les entoure. Ils font partie d'une combinaison qui, seule, permet de les comprendre complètement. La Normandie, grâce à la variété de ses campagnes, en fournit des exemples empruntés à des cadres environnementaux différents.

\section{Les bâtiments d'un village de campagne découverte}

3 La Campagne de Caen se présente comme une plaine à substrat calcaire recouvert de lœss, et où la pluviosité modérée (700 $\mathrm{mm}$ annuels) est favorable à la culture des céréales. Un peuplement précoce en a fait dès l'époque de la Tène un espace agricole fini. Les fermes indigènes dispersées ont été regroupées en villages au XVIII siècle. Le cadre est désormais établi : un finage de champs en lanières, sans autres limites que des bornes enterrées, mais disposé pour permettre des pratiques communautaires, entoure le village où est rassemblée toute la population.

4 Ce village tassé, construit en moellons et pierres de taille calcaires a pour centre l'église (édifiée entre le XII ${ }^{e}$ et le XIV ${ }^{e}$ siècle) et le presbytère (en général reconstruit à la fin du 
XVIII ${ }^{\mathrm{e}}$ siècle). Témoins de la structure foncière en fiefs et de la proximité de Caen, un ou deux châteaux accompagnés d'un parc s'y rencontrent. Les fermes importantes, autrefois propriétés nobles ou bourgeoises, sont à cour fermée et reflètent l'économie céréalière. Le logis fait face au double portail, charretier et piétonnier, et les grange, charretterie, écurie, étable, boulangerie entourent la cour où s'élève parfois le colombier seigneurial. Mais le village comprend aussi les maisons des journaliers, flanquées d'un cellier et d'une remise, et celles des ouvriers agricoles employés aux travaux courants, au battage en grange des céréales puis plus tard au sarclage des plantes industrielles, qui en dépit de leur exiguïté, - une pièce surmontée d'une chambre -, montraient une construction soignée en moellons calcaires assisés et en couvrements en pierre de taille. Les jardins sont ceints de murs de 6 à 9 pieds de haut qui les abritent des vents. Une ou deux mares compensaient la rareté de l'eau sur cette plaine sèche aux vallons parcourus par de rares rivières. La pompe à incendie était abritée à proximité dans un petit bâtiment du début du XIX ${ }^{e}$ siècle. Quand les populations étaient assez importantes on pouvait trouver quelques échoppes (café, épicerie, boulangerie) à la façade caractéristique. Ilot au milieu de la plaine nue, le village apparaissait bien séparé de son finage tout en vivant de lui.

Dans la zone périurbaine qui s'étend jusqu'à 25 kilomètres autour de Caen une évolution profonde est en cours, étrangère à tous les signes qui visaient les relations du village avec son environnement. Dans les lotissements récents les pavillons sont construits en aggloméré crépi et sont séparés les uns des autres par des haies taillées de végétaux exotiques. A leur exemple les nouvelles techniques et matériaux - crépis, peinture des murs, portes et fenêtres standardisés, en bois ou en PVC... - sont peu à peu adoptées dans la partie ancienne et défigurent la modénature des bâtiments. La majorité de la population, travaillant en ville, ressent comme des nuisances les charrois agricoles et les bâtiments agricoles sont rejetés dans la plaine, signes d'un village à l'économie et à la société désormais éclatées (fig. $\left.\mathbf{n}^{\circ} \mathbf{1}\right)\left(\right.$ fig. $\left.\mathbf{n}^{\circ} 2\right)$. 


\section{Figure 1}

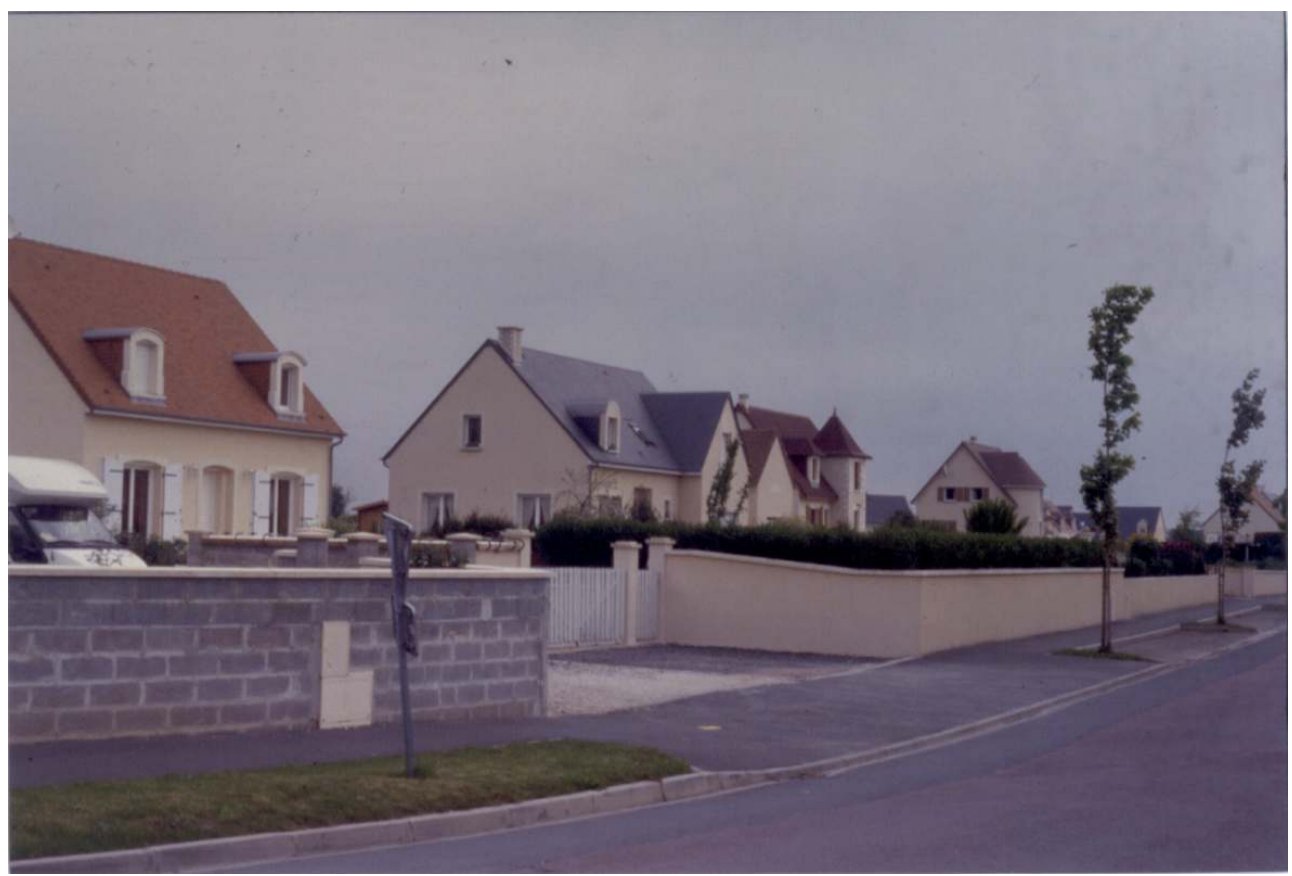

Basly (Calvados). Lotissement récent de maisons à enduit blanc et murets de clôture Phot. P. Brunet ( ) P. Brunet, 2004

Figure 2

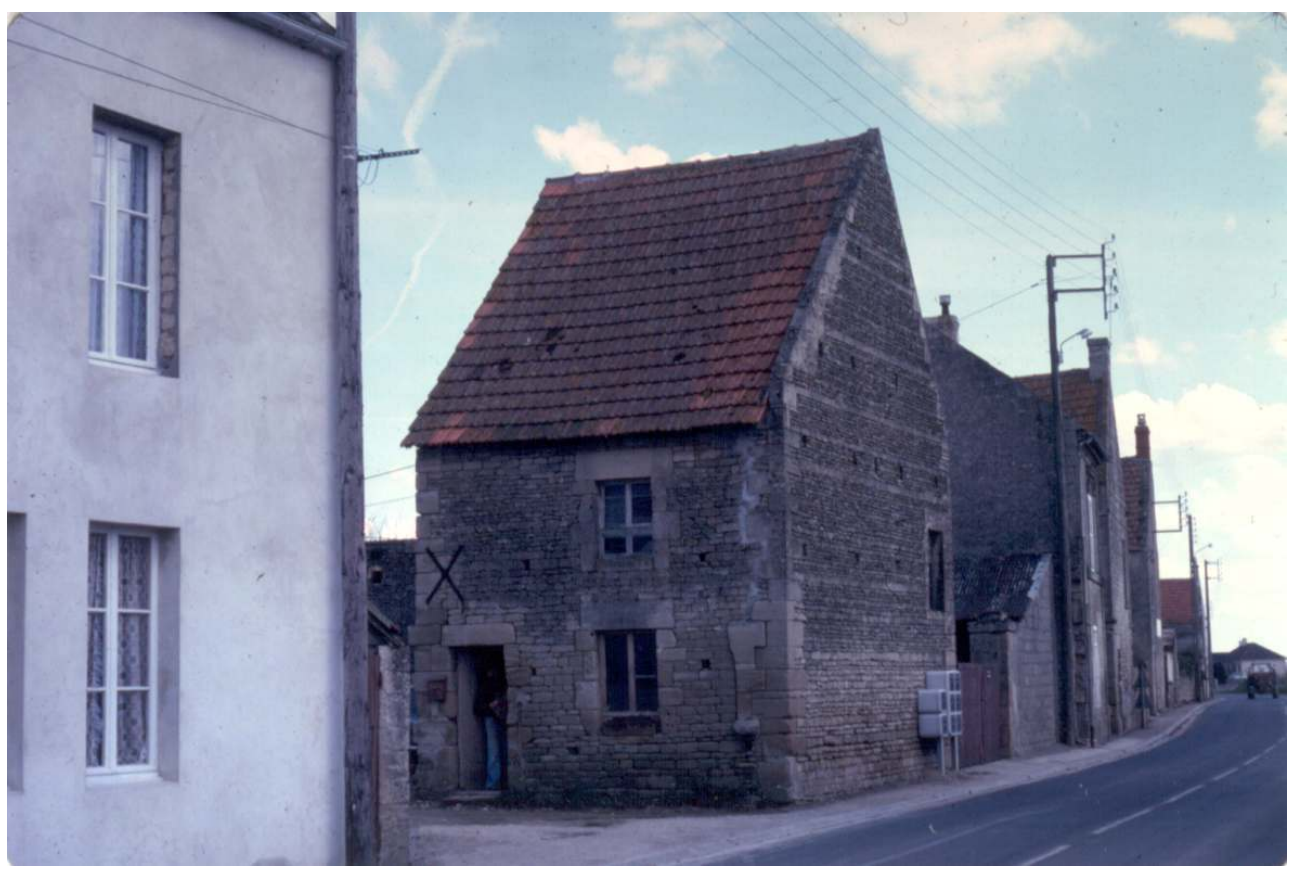

Basly (Calvados). Maison d'ouvrier agricole et murs, avec au premier plan un enduit de ciment qui défıgure l'édifice

Phot. P. Brunet (c) P. Brunet, 2004 


\section{Communes du bocage normand}

6 Dans cette partie occidentale de la Basse-Normandie, un relief plus accidenté et des sols très variés ont offert au peuplement et à la mise en valeur des conditions plus ingrates. La conquête humaine y a été lente et progressive et ne s'est guère épanouie qu'aux XII et XIII ${ }^{\mathrm{e}}$ siècles sous forme de défrichements familiaux. Il en résulte un habitat très dispersé en fermes isolées et en petits hameaux ainsi qu'un paysage d'enclos végétal des parcelles créé à l'origine pour protéger les cultures des divagations du bétail pâturant sur les landes environnantes. La parcelle bâtie, le "plant ", n'échappe pas à ce modèle. Et les exploitations très nombreuses et minuscules qui vivent largement en autarcie $\mathrm{y}$ réunissent à la fois une prairie, un verger de pommiers à cidre et un potager. Sur le " plant » se dispersent de nombreux petits bâtiments : grange, étable, poulailler, four à pain, fagottier, et l'habitation. Si celle-ci est construite en pierre, de nature variable selon les affleurements géologiques locaux (granite, grès, schistes, cornéennes) les bâtiments agricoles sont édifiés en "mâsse » (argile du sol) dans un pan de bois, cadre assez fruste, fourni par les haies. Le chef-lieu paroissial regroupait l'église, la mairie, un café et n'était guère plus important que les autres hameaux. Il y avait donc une grande harmonie entre cet habitat et son environnement.

7 Cette région a subi deux transformations successives. Pendant la première moitié du XX siècle la culture des céréales vivrières a été abandonnée au profit de la prairie, support d'un élevage bovin laitier. Mais les conséquences ont été modérées: les champs sont devenus des herbages dont les haies assuraient la clôture pour le bétail, et les granges ont été remaniées en étables pour des animaux beaucoup plus nombreux.

8 L'évolution du dernier demi-siècle a été plus profonde. De très nombreuses exploitations ont disparu, la moitié par exemple dans le canton de Brécey (Manche) depuis 1870. Les fermes supérieures à 35 hectares qui n'y étaient que 6 sont maintenant 132. En même temps, l'adoption du maïs-fourrage permettait une intensification et un agrandissement des troupeaux. Cette culture mécanisée a poussé au remembrement des terres avec la suppression de nombreuses haies. Matériel agricole et troupeau accrus ont fait éclater le "plant» par la construction en matériaux modernes (planches, tôles) de hangars et de grandes stabulations tandis que disparaissaient les vergers et beaucoup de petits bâtiments très fragiles. Et le "plant » ne subsiste que lorsqu'il est devenu résidence d'employés, d'ouvriers ou de retraités qui y introduisent le jardin d'agrément, les arbres décoratifs, les végétaux exotiques (lauriers, faux-cyprès), et ne conservent que l'habitation et un garage (fig. $\mathbf{n}^{\circ} \mathbf{3}$ ). 
Figure 3

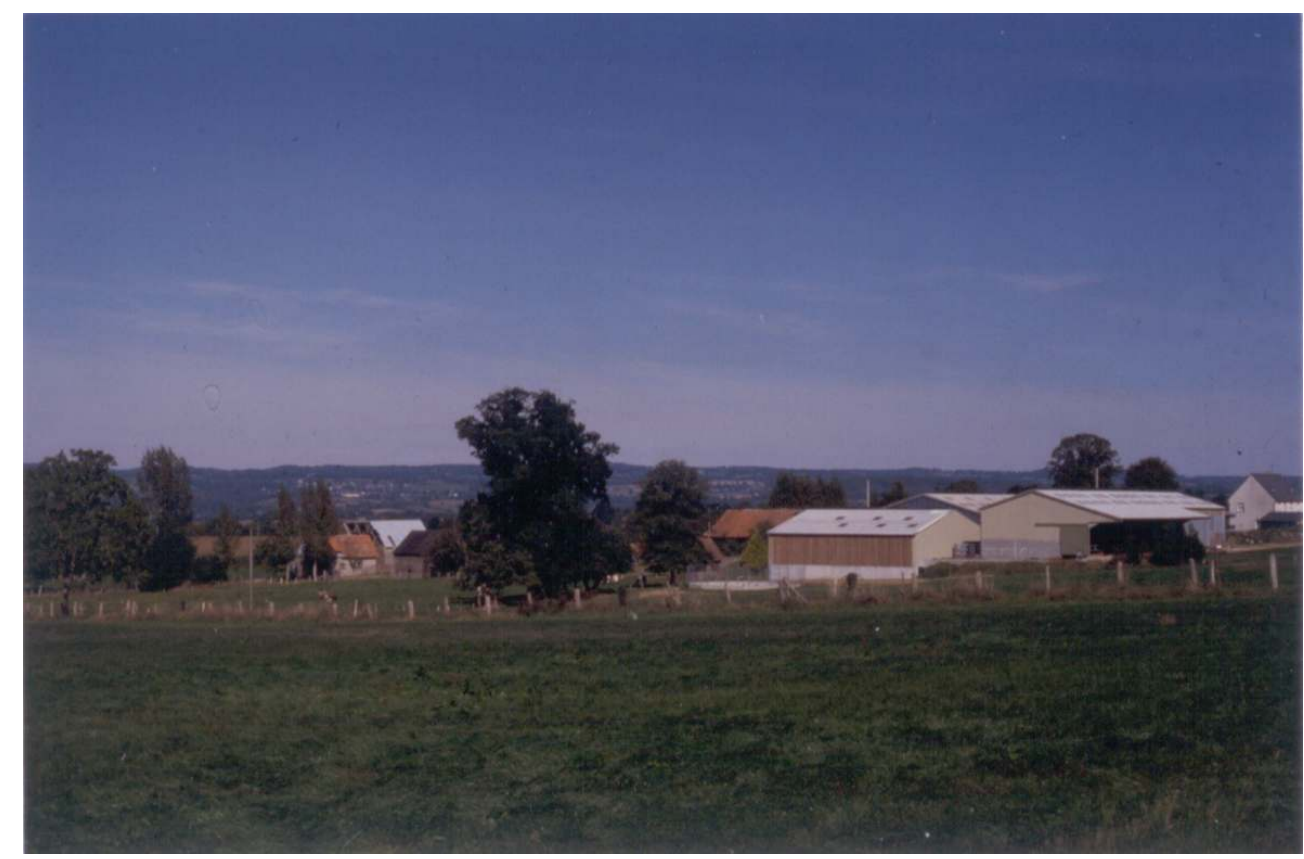

Les Cresnays (Manche). «Plant » ancien à gauche et bâtiments d'élevage récents construits en dehors

Phot. P. Brunet (C) P. Brunet, 2004

9 A l'image de ce paysage transformé, les bâtiments traduisent les changements de la société et de l'économie agricole.

\section{Le Pays de Caux}

10 Ce plateau balayé par les vents d'ouest qui s'avance, tel un promontoire, dans la Manche, offre l'originalité d'associer une campagne découverte à un habitat bocager. L'espace agricole organisé en champs plus ou moins grands est voué aux cultures céréalières, industrielles et fromagères. Les villages et les fermes champêtres isolées sont constitués de «clos-masures ». Le clos-masure est une parcelle de un à quatre hectares, entourée d'un talus surmonté d'une double rangée de grands arbres, plantées de pommiers à cidre et sur laquelle se dispersent les nombreux bâtiments de la ferme. Ainsi s'opposent le monde de l'ombre au monde des grands horizons (fig. $\mathbf{n}^{\circ} \mathbf{4}$ ). 
Figure 4

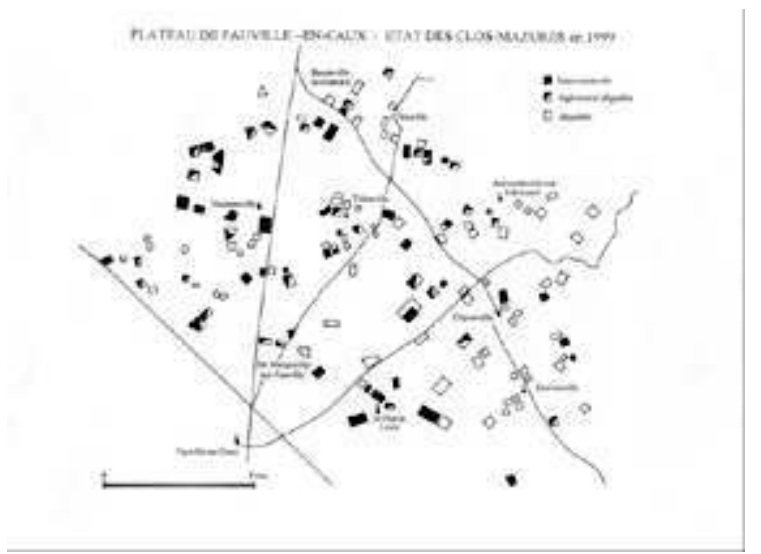

Carte : plateau de Fauville-en-Caux. Etat des clos-masures en 1999

Carte P. Brunet (c) P. Brunet

11 Si l'origine du clos-masure demeure un problème historique non résolu, ses caractères sont en étroit rapport avec son environnement. Les bâtiments agricoles (grange, écurie, étable, charretterie, pressoir, four à pain) sont construits en pan de bois qu'expliquent l'argile ou lœss qui tapisse le plateau et la rareté de la pierre dure de ce pays crayeux. Le silex, parfois utilisé, ou quelques grès sont empruntés à la craie ou à des dépôts superficiels. La brique avec laquelle sont édifiées beaucoup d'habitations depuis le XVII ${ }^{\mathrm{e}}$ siècle, vient aussi de l'argile ou lœss. D'autre part les nombreux bâtiments nécessaires autant aux céréales qu'à l'élevage reflètent un système de polyculture précocement intensifié dès la fin du XVIII ${ }^{\mathrm{e}}$ siècle, grâce à une propriété citadine acquise à partir du XVI e siècle.

Cette relation avec l'environnement est aujourd'hui menacée. La concentration des exploitations supprime la fonction agricole de nombreux clos-masures qui deviennent résidences principales ou secondaires, souvent subdivisées. Une partie des bâtiments disparaissent, même si le pan de bois est à la mode. Des bâtiments en matériaux actuels y sont ajoutés. Les haies, qui furent souvent replantées en hêtres au début du XIX ${ }^{\mathrm{e}}$ siècle, arrivent en fin de vie. Abattues, elles ne sont pas remplacées ou modifiées dans leur silhouette par l'utilisation des peupliers. Les lotissements qui pourraient occuper les grands clos-masures craignent l'entourage de grands arbres. Ainsi s'estompe l'opposition paysagère entre la campagne et l'habitat.

\section{RÉSUMÉS}

En pays de bocage comme en pays d'openfield, l'étude de l'inscription des bâtiments dans le paysage, de leur répartition sur le parcellaire, est essentielle à une bonne compréhension de l'architecture rurale. Plus que toute autre, l'évolution du dernier demi-siècle a entraîné la 
disparition ou la transformation de nombreuses constructions et aménagements, modifiant en profondeur la relation de l'architecture avec l'environnement.

Both in bocage country and in open-field country, the study of the ways buildings are set in the landscape and the ways they are disposed on the plot of property is essential for the correct understanding of rural architecture. More than in earlier periods, the last fifty years have seen the disappearance or the transformation of many buildings and structures, profoundly changing the relation between architecture and the environment.

\section{INDEX}

Keywords : bocage, openfield, rural building, rural architecture, farm, landscape, building materials, agricultural economy, Normandy, Campagne de Caen, Brécey, pays de Caux, agricultural building

Mots-clés : bocage, openfield, bâtiment rural, architecture rurale, ferme, paysage, matériaux de construction, économie agricole, Normandie, Campagne de Caen, Brécey, pays de Caux, bâtiment agricole

\section{AUTEUR}

\section{PIERRE BRUNET}

Professeur émérite de l'Université de Caen 